\title{
CURRENT-CONTROLLED SAWTOOTH GENERATOR
}

\author{
MUHAMMAD TAHER ABUELMA'ATTI* and MUNIR KULAIB ALABSI \\ King Fahd University of Petroleum and Minerals, Box 203, Dhahran 31261, Saudi Arabia
}

(Received 7 June 2003; In final form 28 July 2003)

\begin{abstract}
A simple current-controlled sawtooth generator is presented. Both the amplitude and the frequency of the generated sawtooth can be current-controlled. The circuit uses off-the-shelf components. Experimental results are included.

Keywords: Function generators
\end{abstract}

\section{INTRODUCTION}

Sawtooth and pulse generators find numerous applications in many diverse fields, for example, in instrumentation, measurement and control. In most of the existing sawtooth generators, a grounded capacitor is charged at a pre-specified rate using a constant-current source, and then rapidly discharged through a transistor switch [1-5]. However, while electronic control of the frequency is available in some of the circuits, none of the available circuits can simultaneously provide electronic control of the frequency and the amplitude. The purpose of this article is therefore to present a simple sawtooth generator with electronic control of the frequency and the amplitude.

\section{PROPOSED CIRCUIT}

The proposed circuit is shown in Figure 1. The operation of this circuit can be described as follows:

1. When the voltage at pin 3 is high, that is $V_{\mathrm{CC}}$ the output current of the operational transconductance (OTA1) equals the input auxiliary bias current $I_{\mathrm{b} 1}$. This is attributed to the fact that when the differential input voltage of the OTA is greater than a few millivolts, then all the bias current will be pushed into the output of the OTA. In the meantime, a high voltage at pin 3 will switch off the switch $S_{1}$. Thus, the capacitor $C$ will charge at a constant rate equals $I_{\mathrm{b} 1} / C$.

\footnotetext{
* Corresponding author. E-mail: mtaher@kfupm.edu.sa
} 


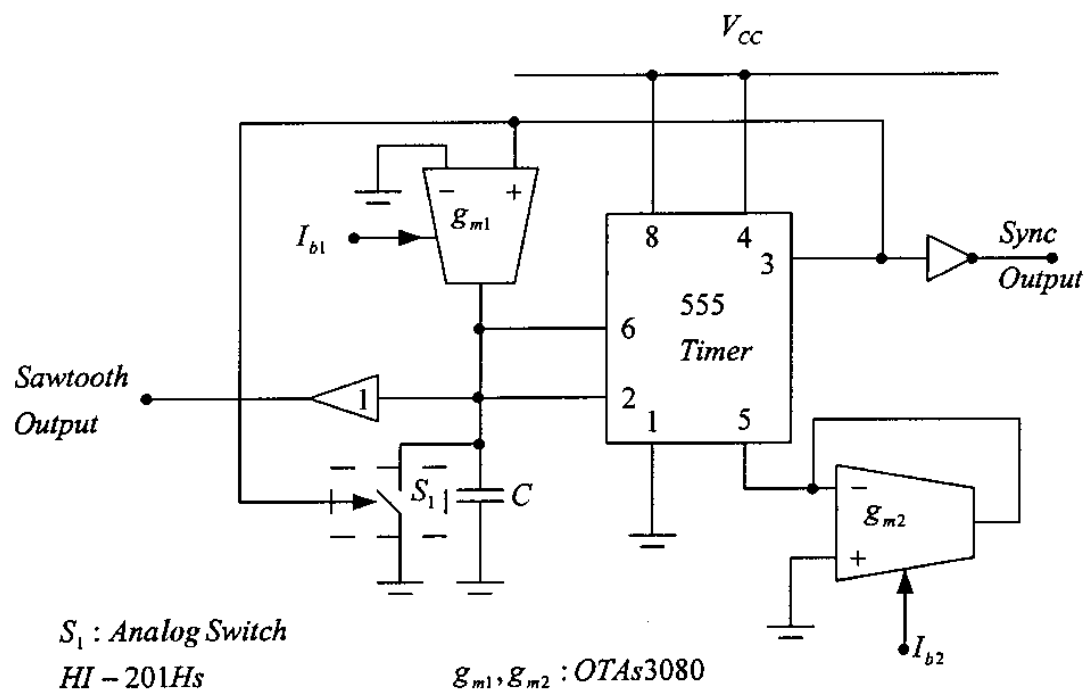

FIGURE 1 Proposed current-controlled sawtooth generator.

2. The charging cycle will terminate when the voltage across the capacitor reaches $V_{5}$, the value of the voltage at pin 5 . The voltage at pin 3 will drop to zero, and the switch $S_{1}$ will close. The capacitor will discharge immediately through the very low resistance of the switch.

3. When the voltage across the capacitor drops below $V_{5} / 2$, the voltage at pin 3 will be high again, switch $S_{1}$ will open and another charging cycle will start.

4. During the charging cycle, the amplitude of the sawtooth will increase from $V_{5} / 2$ to $V_{5}$.

5. Using a standard 555 timer, with three equal resistances each of value $5 \mathrm{k} \Omega$, and assuming that $1 / g_{m 2} \leq 5 \mathrm{k} \Omega$, the voltage at pin 5 can be approximated by $V_{5} \cong V_{\mathrm{CC}} / 5 g_{m 2}$, where $g_{m 2}=I_{\mathrm{b} 2} / 2 V_{\mathrm{T}}$ is the transconductance of OTA2 in $\mathrm{mA} / \mathrm{V}$, and $V_{\mathrm{T}}$ is the thermal voltage $\cong 25 \mathrm{mV}$ at room temperature. Thus the amplitude of the output sawtooth can be expressed as

$$
A \cong \frac{V_{\mathrm{CC}} V_{\mathrm{T}}}{5 I_{\mathrm{b} 2}}
$$

6. Ignoring the discharging time, which is very small, especially for very low values of the switch on-resistance, the frequency of the output sawtooth can be approximated by

$$
f(\mathrm{kHz}) \cong \frac{5 I_{\mathrm{b} 1} I_{\mathrm{b} 2}}{C V_{\mathrm{CC}} V_{\mathrm{T}}}
$$

From Eqs. (1) and (2), it appears that while the frequency of the output sawtooth can be controlled by adjusting the auxiliary bias $I_{\mathrm{b} 1}$ and $I_{\mathrm{b} 2}$, the amplitude can be controlled by adjusting the auxiliary bias current $I_{\mathrm{b} 2}$. Thus, a recommended strategy is to start by adjusting the amplitude using the current $I_{\mathrm{b} 2}$, then adjusting the frequency using the current $I_{\mathrm{b} 1}$.

\section{EXPERIMENTAL RESULTS}

The proposed circuit of Figure 1 was experimentally tested using the CA3080 OTA and the analog switch HI-201HS. This monolithic IC has very low parasitic capacitances and contains four single pole single through (SPST) analog switches with an on-resistance of just 


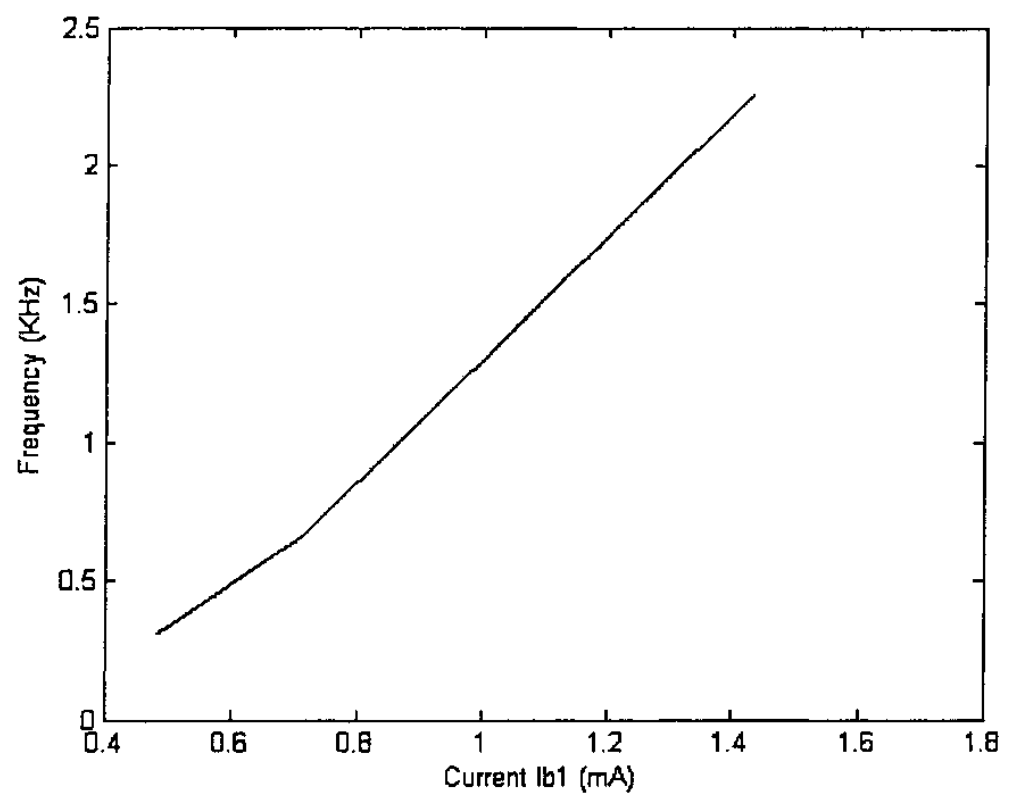

FIGURE 2 Variation of the sawtooth frequency with the control current $I_{\mathrm{b} 1} . V_{\mathrm{CC}}=5 \mathrm{~V}, C=30 \mathrm{nF}, I_{\mathrm{b} 2}=0.7 \mathrm{~mA}$.

$30 \Omega$. Thus, by connecting the four SPST switches in parallel an on-resistance as low as $7.5 \Omega$ can be achieved and high accuracies can be achieved. The sawtooth output is taken from the voltage across the capacitor via a buffer to isolate the load. By inverting the output at pin 3, a pulse with very narrow width, that is a sync, can be obtained.

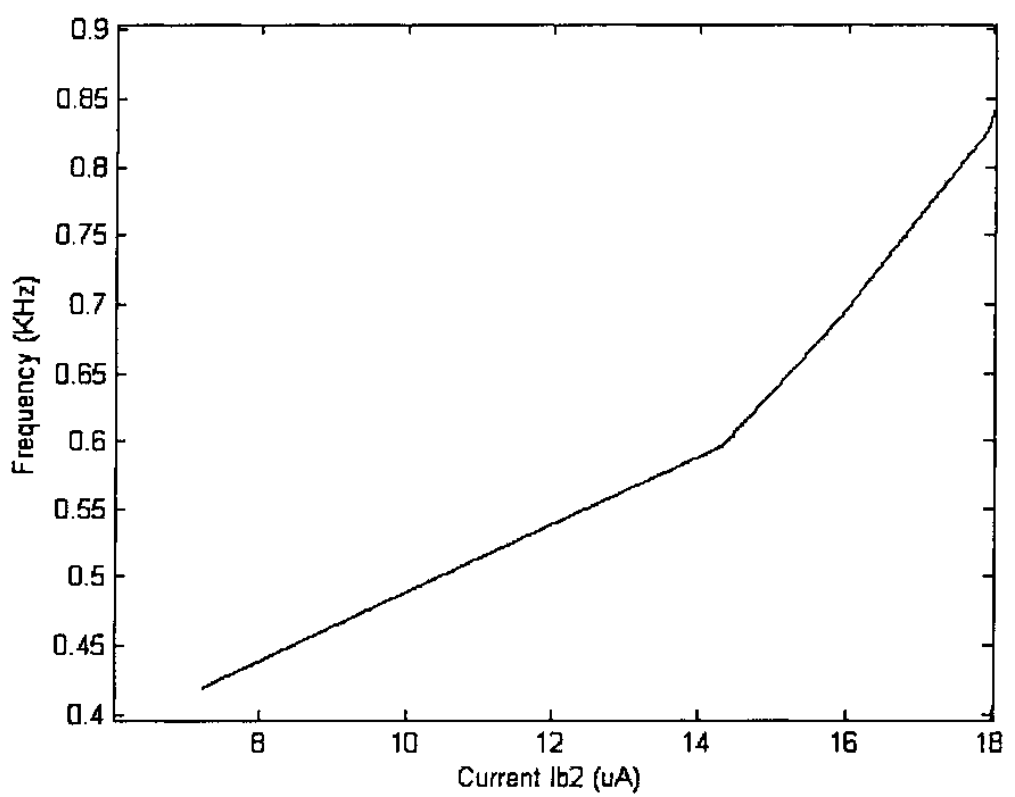

FIGURE 3 Variation of the sawtooth frequency with the control current $I_{\mathrm{b} 2} . V_{\mathrm{CC}}=5 \mathrm{~V}, C=30 \mathrm{nF}, I_{\mathrm{b} 1}=0.7 \mathrm{~mA}$. 


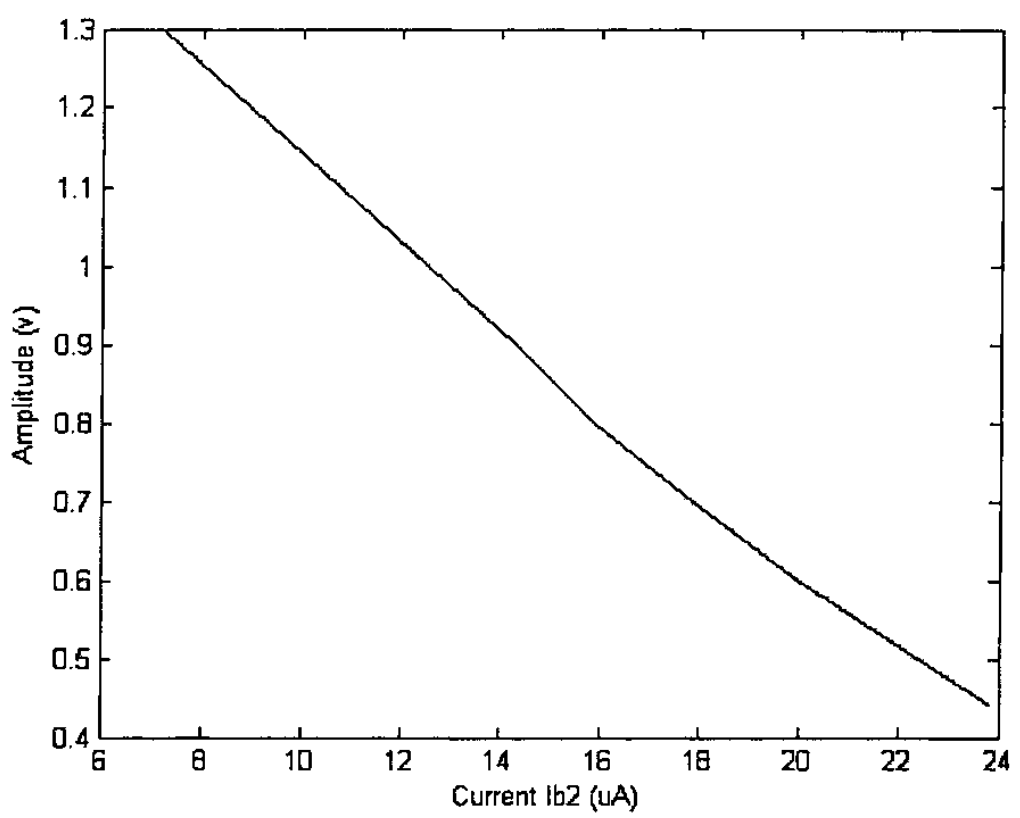

FIGURE 4 Variation of the sawtooth amplitude with the control current $I_{\mathrm{b} 2} . V_{\mathrm{CC}}=5 \mathrm{~V}, C=30 \mathrm{nF}, I_{\mathrm{b} 1}=0.7 \mathrm{~mA}$.

Figures 2 and 3 show the variations of the frequency of oscillation with the bias currents $I_{\mathrm{b} 1}$ and $I_{\mathrm{b} 2}$. These results were obtained with $C=30 \mathrm{nF}$ and $V_{\mathrm{CC}}=5.0 \mathrm{~V}$. Figure 4 shows the variation of the sawtooth amplitude with the bias current $I_{\mathrm{b} 2}$. Figure 5 shows typical output waveforms. It appears from Figures 2-5 that the agreement between the measurements and

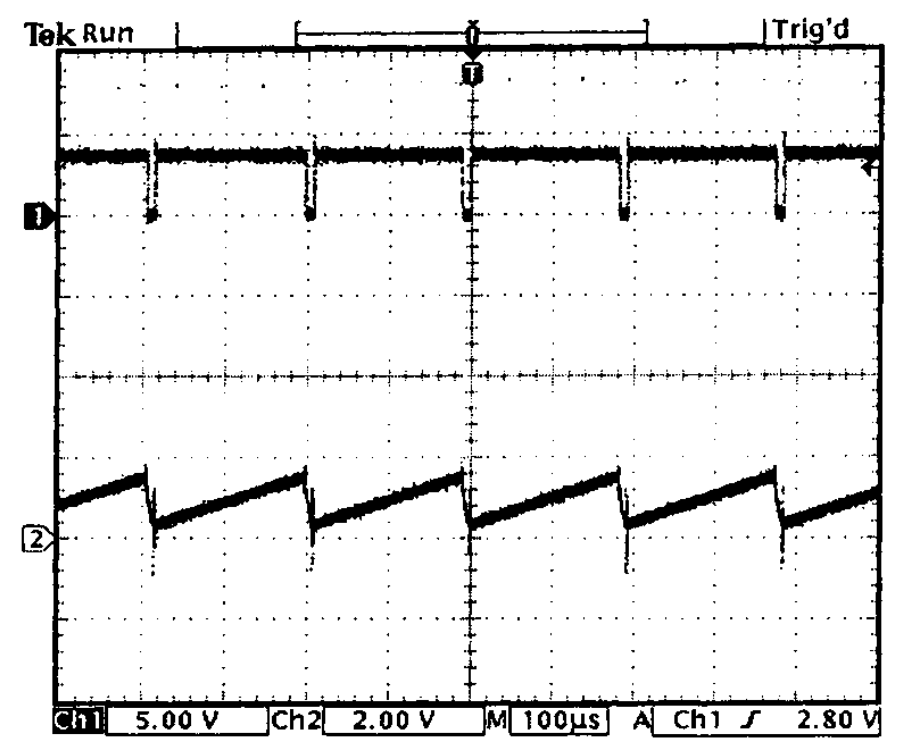

FIGURE 5 Typical output waveforms obtained with $V_{\mathrm{CC}}=5 \mathrm{~V}, C=30 \mathrm{nF}, I_{\mathrm{b} 1}=1.4 \mathrm{~mA}, I_{\mathrm{b} 2}=0.7 \mathrm{~mA}$. 
the presented theory is fairly good. Discrepancies can be attributed to violations of the approximations leading to Eqs. (1) and (2).

\section{CONCLUSION}

A simple current-controlled sawtooth generator has been presented. The circuit is built around the 555 timer and uses a grounded capacitor and two operational transconductance amplifiers to achieve current control of the amplitude and the frequency of the sawtooth waveform. It is worth mentioning here that since the sawtooth output is taken from the capacitor, then any load resistance connected across the capacitor would take its current from the charging current of the capacitor. This may result in distorting the sawtooth output voltage. Such distortion can be avoided by inserting a unity-gain buffer between the capacitor and the load.

\section{References}

[1] Karnal, A., Tripathi, K. C. and Prakash, V. (1984). Inexpensive sawtooth wave; adjustable and frequencyindependent duty-cycle square-wave oscillator. IEEE Trans. Instr. Meas., IM-36, 120-123.

[2] Franco, S. (1988). Design with Operational Amplifiers and Analog Integrated Circuits. McGraw-Hill, New York.

[3] Soclof, S. (1983). Applications of Analog Integrated Circuits. Prentice-Hall, New Jersey.

[4] McMenamin, J. M. (1985). Linear Integrated Circuits. Prentice-Hall, New Jersey.

[5] Abuelma'atti, M. T. and Hassan, F. A. (1985). Novel current(voltage)-controlled linear sawtooth generators. Modelling, Measurement \& Control, 46(3), 47-51. 

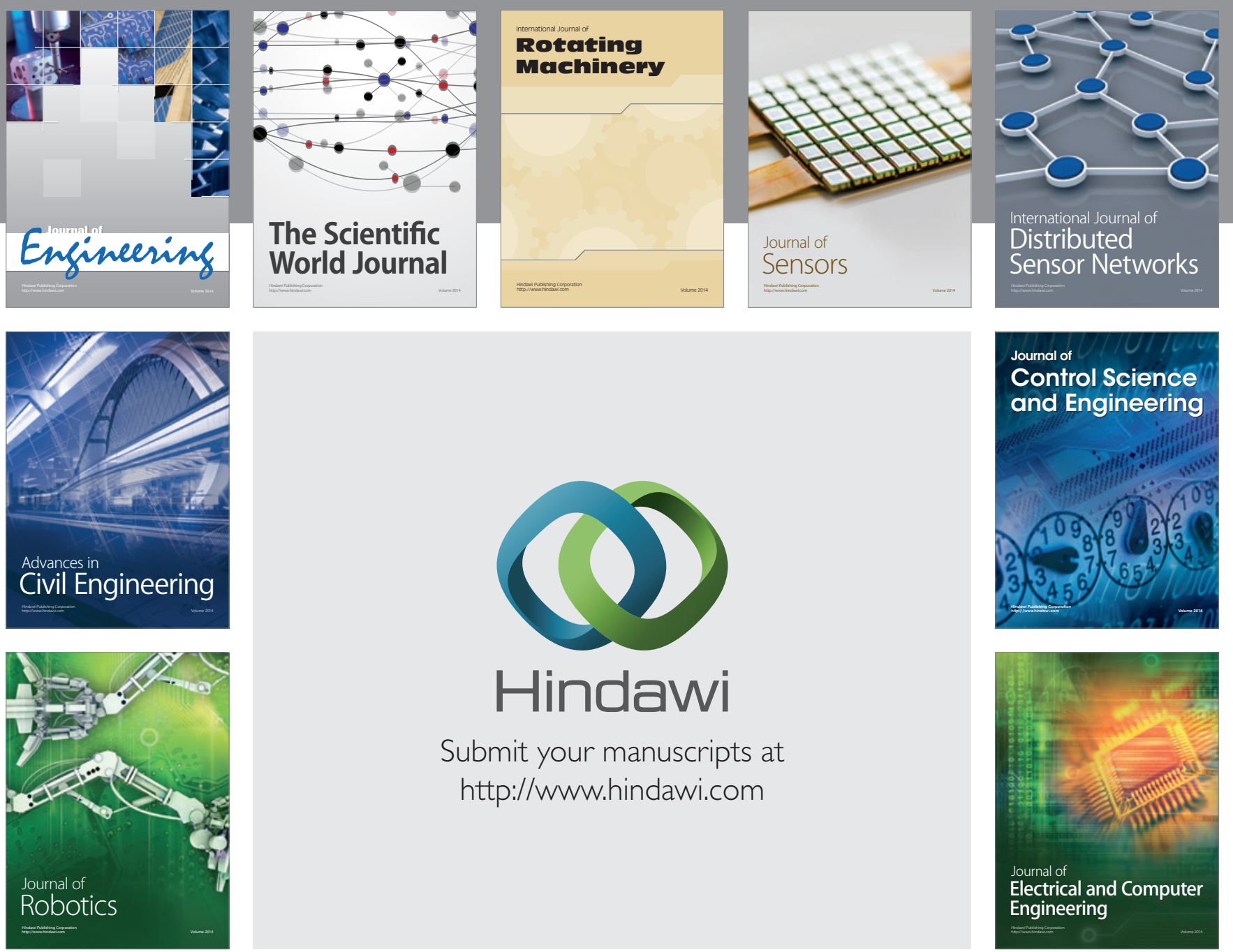

Submit your manuscripts at

http://www.hindawi.com
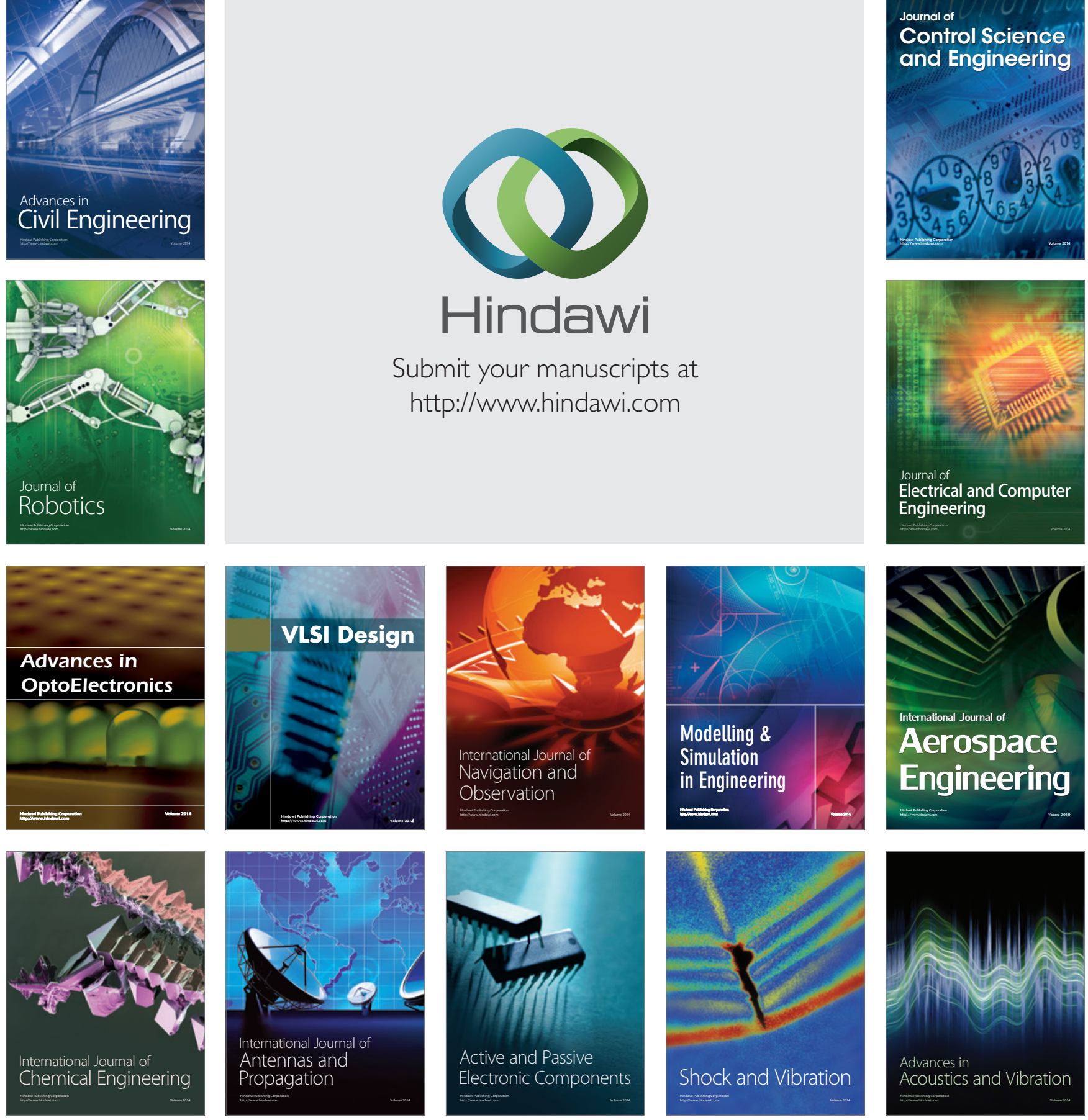